\title{
Pronitridine Nitrification Inhibitor With Urea Ammonium Nitrate for Corn
}

\author{
Kelly A. Nelson ${ }^{1}$ \\ ${ }^{1}$ Division of Plant Sciences, University of Missouri, Novelty, MO, USA \\ Correspondence: Kelly A. Nelson, Lee Greenley Jr. Memorial Research Center, Division of Plant Sciences, \\ University of Missouri, Novelty, MO 63460, USA. Tel: 1-660-739-4410. E-mail: nelsonke@missouri.edu
}

Received: March 5, 2018

doi:10.5539/jas.v10n6p16
Accepted: April 3, $2018 \quad$ Online Published: May 15, 2018

URL: https://doi.org/10.5539/jas.v10n6p16

\begin{abstract}
Nitrification inhibitors have been used to enhance the efficiency of nitrogen fertilizers. This research evaluated the effectiveness of nontreated urea ammonium nitrate (UAN) at $0,67,135,202$, and $270 \mathrm{~kg} \mathrm{~N}^{-1}$ as well as UAN treated with nitrification inhibitors (pronitridine at 9.4 and $18.8 \mathrm{~L} \mathrm{ha}^{-1}$ or nitrapyrin at $0.5 \mathrm{~kg}$ a.i. ha ${ }^{-1}$ ) to enhance $\mathrm{N}$ uptake and increase yield of corn (Zea mays L.). The study took place from 2012-2014 in upstate Missouri on a claypan soil. During the experiments, environmental conditions (high, medium, and low yielding years) affected corn response to pronitridine and nitrapyrin. In general, UAN plus pronitridine at $9.4 \mathrm{~L} \mathrm{ha}^{-1}$ had similar effects on corn compared pronitridine at a higher $\left(18.7 \mathrm{~L} \mathrm{ha}^{-1}\right)$ rate. During a high-yielding year (2014), in order to produce yields equivalent to $67 \mathrm{~kg} \mathrm{~N} \mathrm{ha}^{-1}$ plus pronitridine at $9.4 \mathrm{~L} \mathrm{ha}^{-1}$ or nitrapyrin, UAN needed to be increased 14 to $19 \%$. Similarly, the amount of nontreated UAN needed to be increased 8 to $11 \%$ for yields to be equivalent to UAN at $135 \mathrm{~kg} \mathrm{~N}^{-1}$ plus pronitridine at $9.4 \mathrm{~L} \mathrm{ha}^{-1}$ or nitrapyrin. Grain N removal and agronomic efficiency was highest with pronitridine at $9.4 \mathrm{~L} \mathrm{ha}^{-1}$ and nitrapyrin combined with 67 and $135 \mathrm{~kg} \mathrm{~N}$ $\mathrm{ha}^{-1}$, respectively. This research indicates that pronitridine was as effective as nitrapyrin when added to a pre-emergence application of UAN placed between the rows in a dribble band.
\end{abstract}

Keywords: corn, enhanced efficiency fertilizer, nitrification inhibitor, nitrogen, nitapyrin, pronitridine, urea ammonium nitrate

\section{Introduction}

Nitrogen is a critical input for high-yielding corn production. In 2010, nitrogen applied to corn in the United States totaled over 5 million Mg (USDA-ERS, 2015). More than $40 \%$ of U.S. N consumption is nitrogen solution (USDA-ERS, 2015), of which a majority is urea ammonium nitrate (UAN). Urea ammonium nitrate, which is made by dissolving ammonium nitrate and urea in water, contains $50 \% \mathrm{~N}$ as amide, $25 \%$ as nitrate and $25 \%$ as ammonium. Liquid UAN can be applied in the spring, but it is commonly used for sidedress applications. Nitrogen fertilizer is susceptible to loss, which depends on environmental and field conditions. When soils are saturated for an extended period and conditions are warm, denitrification may be a major loss mechanism especially on poorly drained soils. In such conditions, nitrification inhibitors may benefit farmers. Others have reviewed the development and effects of nitrification inhibitors on reducing $\mathrm{N}$ loss and crop production (Stelly, 1980; Prasad \& Power, 1995; Wolt, 2004; Cook et al., 2015).

Nitrification inhibitors have been utilized not only to reduce gaseous and leaching loss of $\mathrm{N}$ fertilizer by delaying nitrification of $\mathrm{N}$ fertilizers in the soil (Bremner \& Blackmer, 1979; Aulakh et al., 1984; Bronson et al., 1992; Delgado \& Mosier, 1996; Weiske et al., 2001; O’Callaghan et al., 2010; Khalil, 2011; Omode \& Vyn, 2013; Aita et al., 2014; Fisk et al., 2015; Frame, 2017), but also to increase crop yields (Randall et al., 2003; Ruser \& Schulz, 2015; Ren et al., 2017). The most consistent results in the north central U.S. with nitrapyrin (2-chloro-6-(trichloromethyl) pyridine) and dicyandiamide (DCD) were reported on coarse-textured soils with reduced rates of N (Malzer et al., 1989). Extensive research has evaluated how nitrapyrin affects Nitrosomonas in soils as a bactericide with anhydrous ammonia (Hughes \& Welch, 1970; Bremner et al., 1981; Bronson et al., 1992). Nitrapyrin is effective for six to eight weeks in warm soil and can persist longer in cold soils (Trenkel, 2010). Nitrapyrin was marketed in the early 1970's (Prasad \& Power, 1995), and it was recently reformulated as a water-based microencapsulated product (Instinct, Dow AgroSciences, Indianapolis, IN) for use with liquid fertilizer solutions to delay ammonium conversion to nitrate and subsequently reduce the potential for gaseous 
and leaching loss of N (Burzaco et al., 2013; Omonode \& Vyn, 2013; Kyveryga \& Blackmer, 2014). The probability of a yield response to nitrapyrin was greatest when spring precipitation was high (Kyveryga \& Blackmer, 2014). Wolt's (2004) review found that nitrapyrin increased grain yields $7 \%$ and that it reduced leaching loss 16\% and greenhouse gas emissions 51\%. Dicyandiamide was introduced into the U.S. in the 1980's for use with UAN solutions. Nitrosomanas bacteria are suppressed as a bacteriostatic effect with DCD and may stabilize ammonium for 4 to 10 weeks (Mason, 1987; McCarty \& Bremner, 1989; O'Callaghan et al., 2010; Trenkel, 2010; Khalil, 2011).

The Midwestern U.S. has approximately four million ha of claypan soils (Buckley et al., 2010). Waterlogged soil conditions are favorable for denitrification and gaseous N loss (Zurweller et al., 2015; Ren et al., 2017). Claypan soils have a clay layer that is usually less than $50 \mathrm{~cm}$ below the soil surface that causes poor internal drainage (Buckley et al., 2010; Nelson \& Smoot, 2012). These soils are prone to gaseous loss of N fertilizer due to saturated conditions (Nash et al., 2012, 2015; Zurweller et al., 2015). Loss of $\mathrm{N}$ to the atmosphere may approach $30 \%$ (Wilkison et al, 2000). Numerous studies have investigated nitrification inhibitors in soils at high risk of leaching potential, and once $\mathrm{N}$ is leached out of the root zone plant $\mathrm{N}$ uptake is reduced (Mason, 1987; McCarty \& Bremner, 1989; Francis et al., 1993; Martin et al., 1994). A new nitrification inhibitor, pronitridine (CAS RN 1373256-33-7, Centuro ${ }^{\mathrm{TM}}$, Koch Agronomic Services, Witchita, KS), was recently developed to enhance the efficiency of N applications in corn (Vetsch \& Schwab, 2014; Gabrielson \& Epling, 2016). Pronitridine contains a nitrification inhibitor (DCD) plus 30\% N fertilizer (Nitamin Nfusion, Koch Agronomic Services, Witchita, KS) which is a reaction product of ammonia, DCD, formaldehyde, and urea. It was formulated to inhibit nitrification and reduce leaching of the nitrification inhibitor in agriculture crop production systems (Gabrielson \& Epling, 2016). In wet spring conditions in Minnesota, nitapyrin and pronitridine increased corn grain yields 0.8 to 1.1 $\mathrm{Mg} \mathrm{ha}^{-1}$ (Vetsch \& Schwab, 2014). No known research has evaluated corn response to pronitridine with UAN on poorly drained soils. The objective of this research was to evaluate the effectiveness of UAN treated with pronitridine or nitrapyrin nitrification inhibitors to enhance $\mathrm{N}$ uptake and increase corn yield.

\section{Methods}

\subsection{Location}

Field experiments were conducted at the University of Missouri Greenley Research Center near Novelty, MO (40.02324 N, $92.18162 \mathrm{~W}$ ) from 2012 to 2014. The soil was a Putnam silt loam (fine, smectitic, mesic, Vertic Albaqualfs). The study was arranged in a randomized complete block design with five replications in plots 3 by $15 \mathrm{~m}$. This research was arranged as two-factor experiment with five $\mathrm{N}$ rates $\left(0,67,135,202\right.$, and $\left.270 \mathrm{~kg} \mathrm{~N} \mathrm{ha}^{-1}\right)$ and nitrification inhibitors (nontreated, pronitridine (Centuro ${ }^{\mathrm{TM}}$, Koch Agronomic Services, Witchita, KS) at 9.4 $\mathrm{L} \mathrm{ha}^{-1}$, pronitridine at $18.8 \mathrm{~L} \mathrm{ha}^{-1}$, and nitrapyrin (Instinct ${ }^{\circledR}$, Dow AgroSciences, Indianapolis, IN) at $0.5 \mathrm{~kg}$ a.i. $\mathrm{ha}^{-1}$ ). UAN rates were adjusted to offset pronitridine's $\mathrm{N}$ contribution so the total amount of $\mathrm{N}$ applied was the same. Fertilizer was applied pre-emergence by dribble banding between rows with a $\mathrm{CO}_{2}$-propelled hand sprayer. Nitrapyrin may affect nitrification up to $10 \mathrm{~cm}$ from the band (Omonode \& Vyn, 2013). Selected management practices are reported in Table 1.

\subsection{Soil Sampling and Field Measurements}

Before applying fertilizer, composite soil samples were taken from the plot area from each replication using a Uhland probe from four depths $(0-15 \mathrm{~cm}, 16-30 \mathrm{~cm}$, and 31-46 cm). Soil properties, presented by year in Table 2, were analyzed using standard soil testing analytical procedures for Missouri (Nathan et al., 2006). The 0-15 cm sample was analyzed for $\mathrm{pH}\left(0.01 \mathrm{M} \mathrm{CaCl}_{2}\right)$, organic matter content, cation exchange capacity (CEC), available $\mathrm{P}$ (Bray 1-P), and extractable $\left(1 \mathrm{M} \mathrm{NH}_{4} \mathrm{AOc}\right) \mathrm{K}, \mathrm{Ca}$, and $\mathrm{Mg}$. Soil samples from all depths were analyzed for nitrate and exchangeable ammonium concentrations. Precipitation was collected on site (Table 3) throughout the growing season using an automated weather station (Campbell Scientific, Inc., Logan, UT).

Chlorophyll meter readings for 10 ear leaves plot $^{-1}$ were collected to determine $\mathrm{N}$ deficiency (Zhang et al., 2008) using a Minolta SPAD-502 (Konica Minolta Optics, Inc.) at VT (Abendroth et al., 2011). Ear leaves (10 plot ${ }^{-1}$ ) were collected at R1, dried, and analyzed for total $\mathrm{N}$ concentration by combustion using a total $\mathrm{C}: \mathrm{N}$ analyzer (LECO, TruSPEC CN Analyzer, St. Joseph, MI). In 2012 and 2013, ear leaf N concentration and SPAD meter readings were determined prior to the onset of drought conditions (USDM, 2015).

Plant populations were determined prior to harvest. Grain yield, moisture, and test weight were determined using a Wintersteiger Delta (Salt Lake City, UT) equipped with a HarvestMaster GrainGage (SBDS800, Juniper Systems Inc., Logan, UT). Grain yields were adjusted to $150 \mathrm{~g} \mathrm{~kg}^{-1}$ prior to subjecting data to ANOVA. Grain samples were collected and analyzed for $\mathrm{N}$ concentration by combustion using a total C:N analyzer (LECO, TruSPEC CN Analyzer, St. Joseph, MI). In 2013 and 2014, grain samples were also analyzed for protein, oil, and 
starch concentration with a Foss Infratec 1241 Grain Analyzer (Eden Prairie, MN). Agronomic efficiency was calculated as $\left(\mathrm{Y}-\mathrm{Y}_{0}\right) / \mathrm{F}$ where $\mathrm{Y}=$ grain yield of the harvested portion of corn with nutrient applied, $\mathrm{Y}_{0}=$ grain yield of corn with no nutrient applied, and $\mathrm{F}=$ amount of nutrient applied in order to determine the short-term impact of $\mathrm{N}$ on productivity (Dobermann, 2007; Fixen et al., 2014).

Table 1. Selected management practices and application information in 2012, 2013, and 2014

\begin{tabular}{|c|c|c|c|}
\hline Field information & 2012 & 2013 & 2014 \\
\hline Previous crop & Soybean & Soybean & Soybean \\
\hline Tillage & No-till & No-till & No-till \\
\hline Planting date & 2 April & 2 May & 9 April \\
\hline Hybrid & DKC 62-97VT3 & DKC 62-97VT3 & DKC 62-97VT3 \\
\hline Seeding rate $\left(\right.$ seeds ha $\left.{ }^{-1}\right)$ & 79,000 & 81,500 & 81,500 \\
\hline \multicolumn{4}{|l|}{ Fertilizer application dates } \\
\hline Pre-emergence & 6 Apr. & 7 May & 10 Apr. \\
\hline Maintenance & 12 Apr. & 28 Nov. 2012 & 11 Apr. \\
\hline$\left(\mathrm{N}-\mathrm{P}_{2} \mathrm{O}_{5}-\mathrm{K}_{2} \mathrm{O}\right.$ in $\left.\mathrm{kg} \mathrm{ha}^{-1}\right)$ & $(18-90-135)$ & $(18-90-135)$ & $(22-90-157-22$ S-2.2 Zn) \\
\hline \multicolumn{4}{|l|}{ Crop protection chemicals } \\
\hline Fungicide & $\mathrm{NA}^{\dagger}$ & NA & 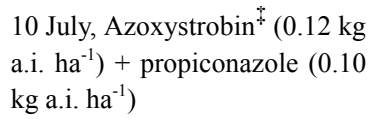 \\
\hline Insecticide & NA & $\begin{array}{l}22 \text { May, Lambda-cyhalothrin } \\
(20 \text { g a.i./ha) }\end{array}$ & NA \\
\hline \multicolumn{4}{|l|}{ Herbicide } \\
\hline Fall & $\begin{array}{l}15 \text { Nov. } 2011 \text {, Simazine }(1.23 \\
\left.\mathrm{kg} \text { a.i. } \mathrm{ha}^{-1}\right)+ \text { glyphosate }(0.53 \\
\left.\mathrm{kg} \text { a.i. } \mathrm{ha}^{-1}\right)+\operatorname{COC}(2.34 \mathrm{~L} \\
\left.\mathrm{ha}^{-1}\right)\end{array}$ & $\begin{array}{l}17 \text { Nov. } 2012 \text {, Simazine }(1.23 \\
\left.\mathrm{kg} \text { a.i. } \mathrm{ha}^{-1}\right)+ \text { glyphosate }(0.53 \\
\left.\mathrm{kg} \text { a.i. } \mathrm{ha}^{-1}\right)+\operatorname{COC}(2.34 \mathrm{~L} \\
\left.\mathrm{ha}^{-1}\right)\end{array}$ & NA \\
\hline POST & $\begin{array}{l}11 \text { May, Acetochlor }(0.94 \mathrm{~kg} \\
\left.\text { a.i. ha }{ }^{-1}\right)+ \text { flumetsulam }(0.03 \\
\left.\mathrm{kg} \mathrm{a.i.}^{-1}\right)+ \text { clopyralid }(0.1 \mathrm{~kg} \\
\left.\text { a.i. } \mathrm{ha}^{-1}\right)+ \text { glyphosate }(1.38 \mathrm{~kg} \\
\left.\text { a.i. ha }{ }^{-1}\right)\end{array}$ & $\begin{array}{l}14 \text { May, Acetochlor }(2.28 \mathrm{~kg} \\
\left.\text { a.i. ha } \mathrm{h}^{-1}\right)+ \text { atrazine }(2.25 \mathrm{~kg} \text { a.i. } \\
\left.\mathrm{ha}^{-1}\right)\end{array}$ & $\begin{array}{l}6 \text { May, Acetochlor }(2.65 \mathrm{~kg} \text { a.i. } \\
\left.\mathrm{ha}^{-1}\right)+ \text { atrazine }(1.88 \mathrm{~kg} \text { a.i. } \\
\left.\mathrm{ha}^{-1}\right)+ \text { glyphosate }(1.06 \mathrm{~kg} \text { a.i. } \\
\left.\mathrm{ha}^{-1}\right)+\operatorname{DAS}\left(0.02 \mathrm{~kg} \mathrm{~L}^{-1}\right)\end{array}$ \\
\hline Late POST & $\begin{array}{l}5 \text { June, Glyphosate }(1.55 \mathrm{~kg} \text { a.i. } \\
\left.\mathrm{ha}^{-1}\right)+ \text { mesotrione }(0.09 \mathrm{~kg} \text { a.i. } \\
\left.\mathrm{ha}^{-1}\right)+ \text { DAS }\left(0.02 \mathrm{~kg} \mathrm{~L}^{-1}\right)+ \\
\operatorname{COC}\left(2.34 \mathrm{~L} \mathrm{ha}^{-1}\right)\end{array}$ & $\begin{array}{l}22 \text { May, Glyphosate }(1.55 \mathrm{~kg} \\
\left.\text { a.i. ha }{ }^{-1}\right)+ \text { mesotrione }(0.09 \mathrm{~kg} \\
\left.\text { a.i. ha }{ }^{-1}\right)+ \text { NIS }(0.25 \% \mathrm{v} / \mathrm{v})+ \\
\text { UAN }\left(2.34 \mathrm{~L} \mathrm{ha}^{-1}\right)\end{array}$ & $\begin{array}{l}11 \text { June, Glyphosate }(1.06 \mathrm{~kg} \\
\left.\text { a.i. } \mathrm{ha}^{-1}\right)+ \text { topramezone }(0.02 \\
\mathrm{kg} \mathrm{a.i.} \mathrm{ha-} \mathrm{ha}^{-1}+\text { atrazine }(0.25 \mathrm{~kg} \\
\left.\text { a.i. ha }{ }^{-1}\right)+ \text { DAS }\left(0.02 \mathrm{~kg} \mathrm{~L}^{-1}\right)\end{array}$ \\
\hline Harvest date & 24 Aug. & 26 Sep. & 6 Oct. \\
\hline
\end{tabular}

Note. ${ }^{\dagger}$ Abbreviations: COC, crop oil concentrate; DAS, Diammonium sulfate; NA, None applied; NIS, nonionic surfactant; POST, postemergence; UAN, urea ammonium nitrate.

${ }^{\ddagger}$ Acetochlor (2-chloro-2'-methyl-6'ethyl-N-ethoxymethylacetanilide); atrazine (2-chloro-4-(ethylamino)-6(isopropylamino)-s-triazine); azoxystrobin, methyl (E)-2-\{2-[6-(2-cyanophenoxy) pyrimidin-4-yloxy]phenyl $\}-3-$ methoxyacrylate; clopyralid, 3,6-dichloro-2-pyridinecarboxylic acid, monoethanolamine salt; diammonium sulfate $\left.\quad\left(\mathrm{NH}_{4}\right)_{2} \mathrm{SO}_{4}\right) ; \quad$ flumetsulam, $\mathrm{N}$-(2,6-difluorophenyl)-5-methyl[1,2,4]triazolo-[1,5-a]pyrimidine-2sulfonamide; glyphosate ( $\mathrm{N}$-(phosphonomethyl)glycine); lambda-cyhalothrin, [1a( $\left.\left.S^{*}\right), 3 \mathrm{a}(\mathrm{Z})\right]-( \pm)$-cyano-(3phenoxyphenyl)methyl-3-(2-chloro-3,3,3-trifl uoro-1-propenyl)-2,2-dimethylcyclopropanecarboxylate; mesotrione (2-[4-(Methylsulfonyl)-2-nitrobenzoyl]cyclohexane-1,3-dione); propiconazole, 1-[[2-(2,4dichlorophenyl)-4-propyl-1,3-dioxolan-2-yl]Methyl]-1H-1,2,4-triazole; and simazine, 2-chloro-4,6bis(ethylamino)-s-triazine; topramezone, [3-(4,5-dihydro-3-isoxazolyl)- 2-methyl-4-(methylsulfonyl)phenyl] (5hydroxy-1-methyl-1H-pyrazol-4-yl) methanone

\subsection{Statistical Analysis}

Data from all years showed a rate response to UAN rates, but the response was affected by the amount and timing of rainfall each year (Table 3). Enhanced-efficiency $\mathrm{N}$ products such as nitrification inhibitors typically perform at rates of $\mathrm{N}$ where loss can be detected in plant measurements and ultimately yield (Frye et al., 1989; Malzer et al., 1989). Therefore, data were sorted by $\mathrm{N}$ rate and subjected to individual ANOVA using PROC 
GLM (SAS, 2014). Nitrification inhibitor means were separated using Fisher's Protected LSD $(P=0.1)$ to determine differences among nitrification inhibitor treatments at specific $\mathrm{N}$ rates. In the absence of a significant interaction, data were combined over years. Quadratic regression analysis (Cerrato \& Blackmer, 1990) was performed using best-fit analysis determined with SigmaPlot (Vers. 8.02, SPSS Inc., Chicago, IL), and significance was determined using SAS (2014). A linear regression analysis evaluated the relationship between grain N and protein concentration, and significance was determined using SAS (2014).

Table 2. Soil test information from 0 to $15 \mathrm{~cm}$ in 2012, 2013, and 2014. Soil nitrate $\left(\mathrm{NO}_{3}-\mathrm{N}\right)$ and ammonium $\left(\mathrm{NH}_{4}-\mathrm{N}\right)$ concentration at three depths on 6 April 2012, 1 May 2013, and 9 April 2014

\begin{tabular}{|c|c|c|c|c|}
\hline Soil test information & Soil depth & 2012 & 2013 & 2014 \\
\hline $\mathrm{pH}\left(0.01 \mathrm{M} \mathrm{CaCl}_{2}\right)$ & $0-15 \mathrm{~cm}$ & $5.9 \pm 0.2^{\dagger}$ & $6.1 \pm 0.1$ & $6.0 \pm 0.2$ \\
\hline Bray 1-P $\left(\mathrm{kg} \mathrm{ha}^{-1}\right)$ & $0-15 \mathrm{~cm}$ & $29.9 \pm 21.1$ & $72.6 \pm 13.1$ & $40.0 \pm 5.2$ \\
\hline $\mathrm{K}\left(\mathrm{kg} \mathrm{ha}^{-1}\right)$ & $0-15 \mathrm{~cm}$ & $228 \pm 31$ & $430 \pm 38$ & $228 \pm 53$ \\
\hline $\mathrm{Ca}\left(\mathrm{kg} \mathrm{ha}^{-1}\right)$ & $0-15 \mathrm{~cm}$ & $4834 \pm 307$ & $4753 \pm 438$ & $4026 \pm 726$ \\
\hline $\operatorname{Mg}\left(\mathrm{kg} \mathrm{ha}^{-1}\right)$ & $0-15 \mathrm{~cm}$ & $585 \pm 94$ & $573 \pm 56$ & $453 \pm 99$ \\
\hline $\operatorname{CEC}\left(\mathrm{cmol}_{\mathrm{c}} \mathrm{kg}^{-1}\right)^{\ddagger}$ & $0-15 \mathrm{~cm}$ & $14.9 \pm 1.3$ & $14.8 \pm 1.2$ & $12.4 \pm 1.8$ \\
\hline $\mathrm{OM}\left(\mathrm{g} \mathrm{kg}^{-1}\right)$ & $0-15 \mathrm{~cm}$ & $33.0 \pm 2.9$ & $31.8 \pm 1.5$ & $26.2 \pm 1.9$ \\
\hline $\mathrm{NO}_{3}-\mathrm{N}\left(\mathrm{mg} \mathrm{kg}^{-1}\right)$ & $0-15 \mathrm{~cm}$ & $9.5 \pm 1.5$ & $16.1 \pm 2.8$ & $15.3 \pm 5.7$ \\
\hline \multirow{5}{*}{$\mathrm{NH}_{4}-\mathrm{N}\left(\mathrm{mg} \mathrm{kg}^{-1}\right)$} & $16-30 \mathrm{~cm}$ & $4.9 \pm 0.4$ & $7.7 \pm 1.1$ & $9.2 \pm 2.1$ \\
\hline & $31-46 \mathrm{~cm}$ & $5.0 \pm 0.5$ & $8.0 \pm 1.3$ & $7.6 \pm 1.7$ \\
\hline & $0-15 \mathrm{~cm}$ & $3.9 \pm 0.3$ & $4.5 \pm 1.1$ & $3.1 \pm 0.5$ \\
\hline & $16-30 \mathrm{~cm}$ & $3.7 \pm 0.7$ & $5.0 \pm 1.4$ & $5.2 \pm 0.8$ \\
\hline & $31-46 \mathrm{~cm}$ & $4.5 \pm 0.7$ & $7.9 \pm 1.8$ & $6.2 \pm 0.9$ \\
\hline
\end{tabular}

Note. ${ }^{\dagger}$ Standard deviation.

* Abbreviations: CEC, cation exchange capacity; OM, organic matter.

\section{Results and Discussion}

\subsection{Precipitation}

The first year of this research was classified as an extreme drought (2012), and the second a severe drought (2013) (USDM, 2015). Precipitation was nearly $200 \mathrm{~mm}$ below average, primarily during grain fill in July (Table 3 ), which reduced overall yields $\left(<3.1 \mathrm{Mg} \mathrm{ha}^{-1}\right)$ in 2012 (Figure 1). In 2013, temperatures during pollination were favorable (data not presented) for high yield potential, but only $48 \mathrm{~mm}$ of rain fell in July and none in August (Table 3), which resulted in small seed size (visual observation). In 2014, precipitation (Table 3) and air temperature (data not presented) through the summer months were favorable for high yields. Although claypan soils are highly productive, they can be susceptible to extreme weather conditions that limit yield (Nelson \& Smoot, 2012; Buckley et al., 2010). The maximum corn yields in the absence of a nitrification inhibitor were determined to be at $166 \mathrm{~kg} \mathrm{~N} \mathrm{ha}^{-1}$ in 2012, $248 \mathrm{~kg} \mathrm{~N} \mathrm{ha}^{-1}$ in 2013, and $237 \mathrm{~kg} \mathrm{~N} \mathrm{ha}^{-1}$ in 2014 (Figure 1), which was affected by precipitation during the growing season (Table 3 ).

Table 3. Monthly precipitation average (10-year) and precipitation during the growing season at Novelty in 2012, 2013, and 2014

\begin{tabular}{|c|c|c|c|c|}
\hline Month & 10 -year average ${ }^{\dagger}$ & 2012 & 2013 & 2014 \\
\hline Apr. & 104 & 119 & 194 & 106 \\
\hline May & 134 & 63 & 261 & 26 \\
\hline June & 133 & 57 & 92 & 225 \\
\hline July & 109 & 19 & 48 & 51 \\
\hline Aug. & 110 & 76 & 0 & 164 \\
\hline Sep. & 90 & 90 & 79 & 175 \\
\hline Total & 680 & 483 & 674 & 747 \\
\hline
\end{tabular}

Note. ${ }^{\dagger}$ Averaged from 2000 to 2011. 


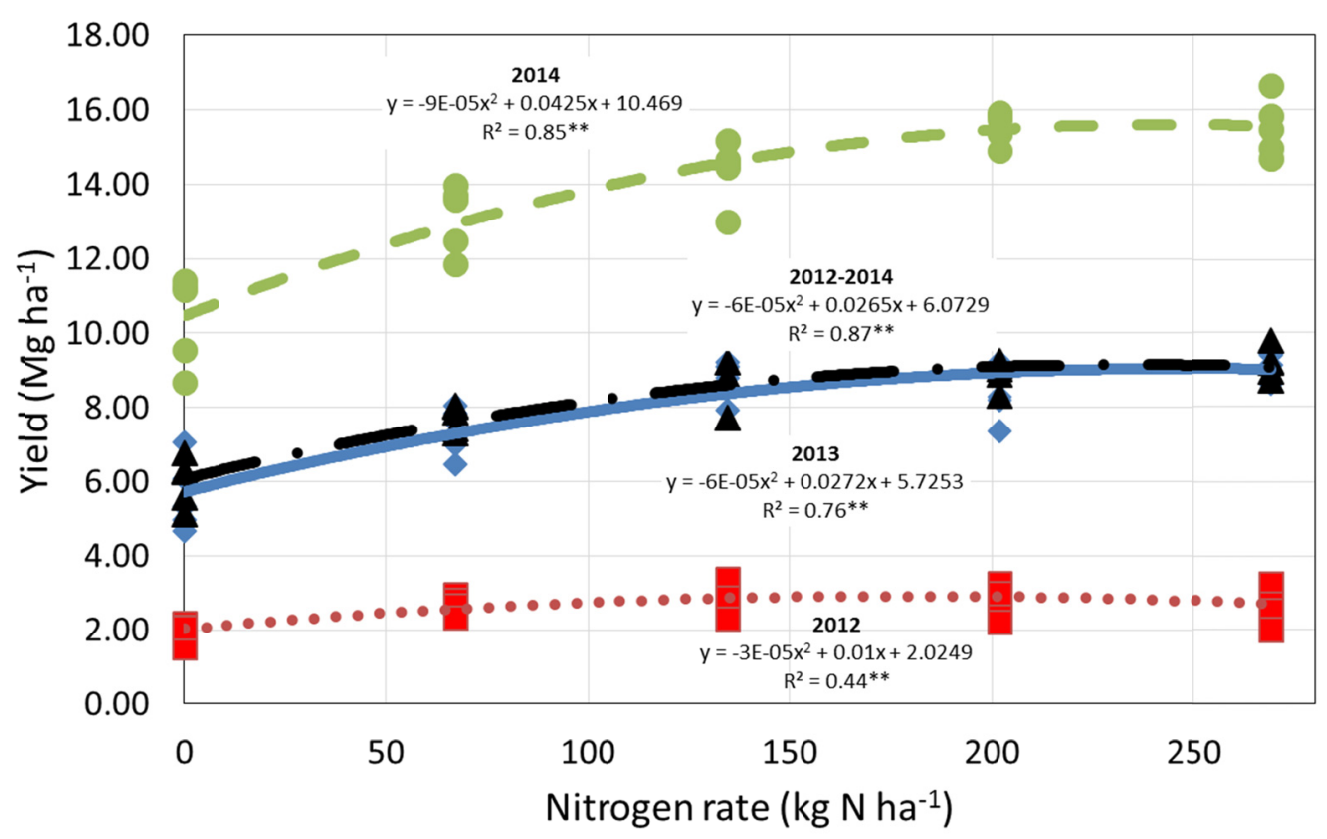

Figure 1. Corn yield response to $\mathrm{N}$ rates in the absence of a nitrification inhibitor in 2012 (dotted line), 2013 (solid line), 2014 (dashed line) and the average (dash-dot line) from 2012-2014 at Novelty, Missouri. Individual points represent plot data from each replication represented in each year

\subsection{UAN at $67 \mathrm{~kg} \mathrm{~N} \mathrm{ha-1}$}

When $\mathrm{N}$ was applied at $67 \mathrm{~kg} \mathrm{~N}$ ha $^{-1}$, no difference in $\operatorname{SPAD}(P=0.29)$, ear leaf $\mathrm{N}$ concentration $(P=0.29)$, plant population $(P=0.62)$, grain moisture $(P=0.74)$, test weight $(P=0.82)$, yield $(P=0.40)$, grain $\mathrm{N}$ concentration $(P=0.76)$, agronomic efficiency $(P=0.76)$, protein $(P=0.93)$, oil $(P=0.87)$, or starch $(P=0.92)$ concentration was observed among the nitrification inhibitors (Table 4). Corn grain yield with UAN at $67 \mathrm{~kg} \mathrm{~N}^{-1} \mathrm{pa}^{-1}$ plus nitrapyrin was $7.98 \mathrm{Mg} \mathrm{ha}^{-1}$ and with pronitridine at $9.4 \mathrm{~L} \mathrm{ha}^{-1}$ was $7.89 \mathrm{Mg} \mathrm{ha}^{-1}$. In order to obtain the same yield with nontreated urea (Figure 1), rates would have to be increased to $78 \mathrm{~kg} \mathrm{~N}^{-1}$ (14\%) to be equivalent to pronitridine at $9.4 \mathrm{~L} \mathrm{ha}^{-1}$ and to $83 \mathrm{~kg} \mathrm{~N} \mathrm{ha}^{-1}(19 \%)$ to be equivalent to nitrapyrin. Increased grain yields with nitrification inhibitors were typically observed at the lower end of a rate response, which was similar to other research (Frye et al., 1989; Malzer et al., 1989). Grain N concentration was higher in 2012 than in 2013 or 2014. The greatest grain $\mathrm{N}$ concentration was observed in the nontreated control, which probably was due to 2012's extreme drought conditions (USDM, 2015). However, grain N concentration was greatest with pronitridine at 9.4 $\mathrm{L} \mathrm{ha}^{-1}$ in 2013 and pronitridine at $18.7 \mathrm{~L} \mathrm{ha}^{-1}$ in 2014 under higher yielding environments. No difference between treatments was observed in 2014. No effects have been reported of DCD or nitrapyrin on corn seed germination (Pal et al., 2016), which was consistent for all of the $\mathrm{N}$ rates evaluated (Tables 4-6). This was expected because nitrogen was dribble banded between the corn rows.

\subsection{UAN at $135 \mathrm{~kg} \mathrm{Nha}^{-1}$}

When the $\mathrm{N}$ amount was $135 \mathrm{~kg} \mathrm{~N}^{-1}$, there was no difference in SPAD values $(P=0.66)$, plant population $(P=$ $0.27)$, grain moisture $(P=0.93)$, test weight $(P=0.50)$, grain $\mathrm{N}$ concentration $(P=0.92)$, grain $\mathrm{N}$ removal $(P=$ $0.82)$, agronomic efficiency $(P=0.82)$, protein $(P=0.33)$, oil $(P=0.40)$, or starch $(P=0.36)$ concentration among treatments (Table 5). Ear leaf $\mathrm{N}$ concentration was ranked nontreated control $\left(26.55 \mathrm{~g} \mathrm{~kg}^{-1}\right)=$ nitrapyrin $\left(26.03 \mathrm{~g} \mathrm{~kg}^{-1}\right) \geq$ pronitridine at $18.8 \mathrm{~L} \mathrm{ha}^{-1}\left(25.50 \mathrm{~g} \mathrm{~kg}^{-1}\right)=$ pronitridine at $9.4 \mathrm{~L} \mathrm{ha}^{-1}\left(24.76 \mathrm{~g} \mathrm{~kg}^{-1}\right)$. This indicated no advantage of a nitrification inhibitor at VT when ear leaf $\mathrm{N}$ concentration was determined. In the extreme drought conditions of 2012, grain yields for nitrification inhibitors were similar (USDM, 2015). Spring conditions were favorable for gaseous $\mathrm{N}$ loss $\left(\mathrm{N}_{2} \mathrm{O}\right.$ and $\left.\mathrm{NH}_{3}\right)$ following fertilizer applications, as reported by Nash et al. (2015) in a separate experiment on site. Claypan soils that have been waterlogged for one week had greater $\mathrm{N}_{2} \mathrm{O}$ emissions than soils with no waterlogging (Zurweller et al., 2015). Excessive precipitation or temperature following application may have affected leaching or decomposition of DCD or nitrapyrin (Bronson et al., 1989; McCarty \& Bremner, 1989); however, the pronitridine formulation may have reduced the leaching 
potential compared to DCD (Gabrielson \& Epling, 2016). Grain yields were greatest in the nontreated control $\left(8.71 \mathrm{Mg} \mathrm{ha}^{-1}\right)$ and were similar to pronitridine at $18.8 \mathrm{~L} \mathrm{ha}^{-1}$ and nitrapyrin in 2013 during a moderately yielding year when $\mathrm{N}$ was probably not limiting due to summer drought conditions. In the high-yield environment of 2014, nitrapyrin and pronitridine at $9.4 \mathrm{~L} \mathrm{ha}^{-1}$ increased yields 0.44 to $0.5 \mathrm{Mg} \mathrm{ha}^{-1}$ compared to the nontreated control at $135 \mathrm{~kg} \mathrm{~N} \mathrm{ha}^{-1}$, which indicated the benefit of a nitrification inhibitor in such production environments when $\mathrm{N}$ rates were reduced. This was similar to other research when a reduced rate of UAN was applied with nitrapyrin (Habibullah et al., 2017). In 2014, nontreated UAN amounts (Table 5) needed to be increased to 147 $\mathrm{kg} \mathrm{N} \mathrm{ha}^{-1}(8 \%)$ and $151 \mathrm{~kg} \mathrm{~N} \mathrm{ha}^{-1}(11 \%)$ in order to obtain yields similar to pronitridine at $9.4 \mathrm{~L} \mathrm{ha}^{-1}$ and nitrapyrin, respectively (Figure 1). The increase in the yield may be due reduced gaseous $\mathrm{N}$ loss in the presence of the nitrification inhibitors (Bronson et al., 1992; Zaman et al., 2009; Chen et al., 2010; Halvorson et al., 2010; Carneiro et al., 2010; Khalil, 2011; Di \& Cameron, 2012; Burzaco et al., 2013; Omonode \& Vyn 2013). This could be because spring conditions generally were wet (Table 3), gaseous $\mathrm{N}$ losses in claypan soils as high as $30 \%$ have been reported (Wilkison et al., 2000), and summer conditions were favorable for high yields. Similar effects between nitrapyrin and pronitridine were expected as Martin et al., (1994) reported finding similar effects of nitrapyrin and DCD on total inorganic soil N.

Table 4. Corn response to nitrification inhibitors with urea ammonium nitrate when $\mathrm{N}$ amount was $67 \mathrm{~kg} \mathrm{~N} \mathrm{ha}^{-1}$. Data were combined over years in the absence of a significant interaction

\begin{tabular}{|c|c|c|c|c|c|c|c|c|c|c|c|c|c|c|}
\hline \multirow{2}{*}{$\begin{array}{l}\text { Nitrification } \\
\text { inhibitor }\end{array}$} & \multirow{2}{*}{ SPAD } & \multirow{2}{*}{$\begin{array}{l}\text { Ear } \\
\text { leaf } N\end{array}$} & \multirow{2}{*}{ Population } & \multirow{2}{*}{ Moisture } & \multirow{2}{*}{$\begin{array}{l}\text { Test } \\
\text { weight }\end{array}$} & \multirow{2}{*}{ Yield } & \multicolumn{3}{|c|}{ Grain N } & \multirow{2}{*}{$\begin{array}{l}\text { Grain } \mathrm{N} \\
\text { removal }\end{array}$} & \multirow{2}{*}{$\begin{array}{l}\text { Agronomic } \\
\text { efficiency }^{\dagger}\end{array}$} & \multirow{2}{*}{ Protein } & \multirow{2}{*}{$\mathrm{Oil}^{\ddagger}$} & \multirow{2}{*}{$\operatorname{Starch}^{+}$} \\
\hline & & & & & & & 2012 & 2013 & 2014 & & & & & \\
\hline & & $\mathrm{g} \mathrm{kg}^{-1}$ & No. ha ${ }^{-1}$ & $\mathrm{~g} \mathrm{~kg}^{-1}$ & $\mathrm{~kg} \mathrm{hL}^{-1}$ & $\mathrm{Mg} \mathrm{ha}^{-1}$ & ------ & $\mathrm{g} \mathrm{kg}^{-1}$ & ------ & $\mathrm{kg} \mathrm{ha}^{-1}$ & kg kg app..$^{-1}$ & $\mathrm{~g} \mathrm{~kg}^{-1}$ & $\mathrm{~g} \mathrm{~kg}^{-1}$ & $\mathrm{~g} \mathrm{~kg}^{-1}$ \\
\hline Non-treated & 49.4 & 23.9 & 72,620 & 177 & 71.6 & 7.73 & 12.87 & 10.01 & 10.57 & 82 & 36.0 & 73.0 & 34.1 & 742 \\
\hline $\begin{array}{l}\text { Pronitridine } \\
\text { at } 9.4 \mathrm{~L} \mathrm{ha}^{-1}\end{array}$ & 49.7 & 24.5 & 69,650 & 180 & 71.6 & 7.89 & 12.44 & 11.18 & 10.43 & 86 & 41.6 & 74.1 & 34.2 & 742 \\
\hline $\begin{array}{l}\text { Pronitridine } \\
\text { at } 18.7 \mathrm{~L} \mathrm{ha}^{-1}\end{array}$ & 47.9 & 23.4 & 71,380 & 181 & 71.5 & 7.71 & 12.11 & 9.80 & 11.31 & 84 & 38.9 & 73.3 & 34.8 & 741 \\
\hline Nitrapyrin & 49.0 & 24.0 & 70,150 & 175 & 71.8 & 7.98 & 12.66 & 9.51 & 10.94 & 85 & 40.3 & 73.4 & 34.6 & 741 \\
\hline $\operatorname{LSD}(P=0.1)$ & NS & NS & NS & NS & NS & NS & $----\cdot$ & -0.91 & ------ & NS & NS & NS & NS & NS \\
\hline $\mathrm{P}>\mathrm{F}$ & 0.29 & 0.29 & 0.62 & 0.74 & 0.82 & 0.40 & & 0.04 & & 0.76 & 0.76 & 0.93 & 0.87 & 0.93 \\
\hline
\end{tabular}

Note. ${ }^{\dagger}$ Calculated as: kg grain produced $\mathrm{kg} \mathrm{N}^{-1}$ applied (Fixen et al., 2014).

$¥$ Protein, oil, and starch concentrations were determined in 2013 and 2014.

Table 5. Corn response to nitrification inhibitors with urea ammonium nitrate when $\mathrm{N}$ amount was $135 \mathrm{~kg} \mathrm{~N} \mathrm{ha}^{-1}$. Data were combined over years in the absence of a significant interaction

\begin{tabular}{|c|c|c|c|c|c|c|c|c|c|c|c|c|c|c|}
\hline \multirow{2}{*}{$\begin{array}{l}\text { Nitrification } \\
\text { inhibitor }\end{array}$} & \multirow{2}{*}{ SPAD } & \multirow{2}{*}{$\begin{array}{l}\text { Ear } \\
\text { leaf } \mathrm{N}\end{array}$} & \multirow{2}{*}{ Population } & \multirow{2}{*}{ Moisture } & \multirow{2}{*}{$\begin{array}{l}\text { Test } \\
\text { weight }\end{array}$} & \multicolumn{3}{|c|}{ Yield } & \multirow{2}{*}{ - Grain N } & \multirow{2}{*}{$\begin{array}{l}\text { Grain N } \\
\text { removal }\end{array}$} & \multirow{2}{*}{$\begin{array}{l}\text { Agronomic } \\
\text { efficiency }^{\dagger}\end{array}$} & \multirow{2}{*}{ Protein $^{\ddagger}$} & \multirow{2}{*}{$\mathrm{Oil}^{+}$} & \multirow{2}{*}{ Starch } \\
\hline & & & & & & 2012 & 2013 & 2014 & & & & & & \\
\hline & & $\mathrm{g} \mathrm{kg}^{-1}$ & No. ha ${ }^{-1}$ & $\mathrm{~g} \mathrm{~kg}^{-1}$ & $\mathrm{~kg} \mathrm{hL}^{-1}$ & ------ & $\mathrm{Mg} \mathrm{ha}^{-1}$ & 1 ------ & $\mathrm{g} \mathrm{kg}^{-1}$ & $\mathrm{~kg} \mathrm{ha}^{-1}$ & $\mathrm{~kg} \mathrm{~kg} \mathrm{app.} .^{-1}$ & $\mathrm{~g} \mathrm{~kg}^{-1}$ & $\mathrm{~g} \mathrm{~kg}^{-1}$ & $\mathrm{~g} \mathrm{~kg}^{-1}$ \\
\hline Non-treated & 52.2 & 26.55 & 72,870 & 175 & 72.1 & 2.83 & 8.71 & 14.32 & 11.78 & 98 & 29.8 & 79.7 & 35.1 & 737 \\
\hline $\begin{array}{l}\text { Pronitridine } \\
\text { at } 9.4 \mathrm{~L} \mathrm{ha}^{-1}\end{array}$ & 51.5 & 24.76 & 70,890 & 174 & 72.6 & 3.03 & 7.94 & 14.76 & 11.94 & 100 & 31.0 & 77.9 & 33.3 & 741 \\
\hline $\begin{array}{l}\text { Pronitridine } \\
\text { at } 18.7 \mathrm{~L} \mathrm{ha}^{-1}\end{array}$ & 51.7 & 25.50 & 69,650 & 174 & 72.4 & 2.92 & 8.34 & 14.44 & 11.77 & 99 & 30.0 & 79.5 & 34.4 & 739 \\
\hline Nitrapyrin & 52.1 & 26.03 & 71,140 & 172 & 72.5 & 2.93 & 8.32 & 14.82 & 11.85 & 101 & 31.5 & 80.9 & 34.9 & 737 \\
\hline $\operatorname{LSD}(P=0.1)$ & NS & 1.01 & NS & NS & NS & ----- & -0.44 & ------- & NS & NS & NS & NS & NS & NS \\
\hline $\mathrm{P}>\mathrm{F}$ & 0.66 & 0.03 & 0.27 & 0.93 & 0.50 & & 0.06 & & 0.92 & 0.82 & 0.82 & 0.33 & 0.40 & 0.36 \\
\hline
\end{tabular}

Note. ${ }^{\dagger}$ Calculated as: kg grain produced $\mathrm{kg} \mathrm{N}^{-1}$ applied (Fixen et al., 2014).

* Protein, oil, and starch concentrations were determined in 2013 and 2014.

\subsection{UAN at $202 \mathrm{~kg} \mathrm{Nha}^{-1}$}

Urea ammonium nitrate at $202 \mathrm{~kg} \mathrm{~N}^{-1} \mathrm{a}^{-1}$ ad corn ear leaf SPAD meter readings at R1 that showed UAN treated with nitrapyrin had greener ear leaves than pronitridine at $9.4 \mathrm{~L} \mathrm{ha}^{-1}$, but values were similar to pronitridine at 18.7 $\mathrm{L} \mathrm{ha}^{-1}$ and the nontreated control (Table 5). However, ear leaf $\mathrm{N}$ concentration was greatest $\left(27.2 \mathrm{~g} \mathrm{~kg}^{-1}\right)$ with pronitridine at $9.4 \mathrm{~L} \mathrm{ha}^{-1}$. Nitrification inhibitor treatments had similar plant populations at harvest $(P=$ 
0.99), grain moisture $(P=0.88)$, test weight $(P=0.74)$, grain $\mathrm{N}$ concentration $(P=0.60)$, grain $\mathrm{N}$ removal $(P=$ $0.46)$, agronomic efficiency $(P=0.45)$, protein $(P=0.95)$, oil, $(P=0.79)$, and starch $(P=0.79)$ concentration. Grain yield for the nitrification inhibitors was ranked pronitridine at $9.4 \mathrm{~L} \mathrm{ha}^{-1}\left(9.02 \mathrm{Mg} \mathrm{ha}^{-1}\right)=$ pronitridine at 18.7 $\mathrm{L} \mathrm{ha}^{-1}\left(9.01 \mathrm{Mg} \mathrm{ha}^{-1}\right)=$ non-treated $\left(8.94 \mathrm{Mg} \mathrm{ha}^{-1}\right) \geq$ nitrapyrin $\left(8.63 \mathrm{Mg} \mathrm{ha}^{-1}\right)$. Differences between pronitridine and nitrapyrin might be due to differences in the longevity of these products in the soil or the ability to affect ammonia volatilization (Fox \& Bandel, 1989; Aita et al., 2014; Frame, 2017). Yield data indicate that adequate $\mathrm{N}$ was supplied to the crop for high yields at $202 \mathrm{~kg} \mathrm{~N}^{-1}$ or greater, and no advantage was observed when adding a nitrification inhibitor when compared to the nontreated control.

Table 6. Corn response to nitrification inhibitors with urea ammonium nitrate when $\mathrm{N}$ amount was $202 \mathrm{~kg} \mathrm{~N} \mathrm{ha}^{-1}$. Data were combined over years in the absence of a significant interaction

\begin{tabular}{|c|c|c|c|c|c|c|c|c|c|c|c|c|}
\hline $\begin{array}{l}\text { Nitrification } \\
\text { inhibitor }\end{array}$ & SPAD & $\begin{array}{l}\text { Ear } \\
\text { leaf } \mathrm{N}\end{array}$ & Population & Moisture & $\begin{array}{l}\text { Test } \\
\text { weight }\end{array}$ & Yield & Grain N & $\begin{array}{l}\text { Grain N } \\
\text { removal }\end{array}$ & $\begin{array}{l}\text { Agronomic } \\
\text { efficiency }^{\dagger}\end{array}$ & Protein ${ }^{\ddagger}$ & $\mathrm{Oil}^{+}$ & $\mathrm{Starch}^{\ddagger}$ \\
\hline & & $\mathrm{g} \mathrm{kg}^{-1}$ & No. ha ${ }^{-1}$ & $\mathrm{~g} \mathrm{~kg}^{-1}$ & $\mathrm{~kg} \mathrm{hL}^{-1}$ & $\mathrm{Mg} \mathrm{ha}^{-1}$ & $\mathrm{~g} \mathrm{~kg}^{-1}$ & $\mathrm{~kg} \mathrm{ha}^{-1}$ & kg kg app..$^{-1}$ & $\mathrm{~g} \mathrm{~kg}^{-1}$ & $\mathrm{~g} \mathrm{~kg}^{-1}$ & $\mathrm{~g} \mathrm{~kg}^{-1}$ \\
\hline Nontreated & 54.1 & 26.6 & 71,140 & 178 & 72.5 & 8.94 & 12.32 & 109 & 25.3 & 85.9 & 34.2 & 736 \\
\hline Pronitridine at $9.4 \mathrm{~L} \mathrm{ha}^{-1}$ & 52.6 & 27.3 & 71,140 & 175 & 72.6 & 9.02 & 12.70 & 112 & 26.6 & 85.6 & 34.8 & 733 \\
\hline Pronitridine at $18.7 \mathrm{~L} \mathrm{ha}^{-1}$ & 54.2 & 26.5 & 71,380 & 175 & 72.6 & 9.01 & 12.49 & 111 & 26.3 & 85.2 & 33.7 & 736 \\
\hline Nitrapyrin & 55.5 & 26.2 & 70,890 & 177 & 72.6 & 8.63 & 12.54 & 107 & 24.4 & 86.2 & 34.4 & 735 \\
\hline $\operatorname{LSD}(P=0.1)$ & 1.8 & $\mathrm{NS}$ & NS & NS & NS & 0.33 & NS & NS & NS & NS & NS & NS \\
\hline$P>F$ & 0.07 & 0.49 & 0.99 & 0.88 & 0.74 & 0.09 & 0.60 & 0.46 & 0.45 & 0.95 & 0.79 & 0.79 \\
\hline
\end{tabular}

Note. ${ }^{\dagger}$ Calculated as: $\mathrm{kg}$ grain produced $\mathrm{kg} \mathrm{N}^{-1}$ applied (Fixen et al., 2014).

* Protein, oil, and starch concentrations were determined in 2013 and 2014.

\subsection{UAN at $270 \mathrm{~kg} \mathrm{Nha^{-1 }}$}

Slight differences among nitrification inhibitors were observed for test weight and starch concentration with pronitridine at $9.4 \mathrm{~L} \mathrm{ha}^{-1}$, nitrapyrin, and the nontreated control having similar test weights and starch concentrations when $\mathrm{N}$ was applied at $270 \mathrm{~kg} \mathrm{~N} \mathrm{ha}^{-1}$ (data not presented). All of the corn response parameters that were evaluated were similar among nitrification inhibitors with UAN at $270 \mathrm{~kg} \mathrm{~N} \mathrm{ha}^{-1}$ (data not presented). This amount of $\mathrm{N}$ was excessive to detect differences among nitrification inhibitors, in keeping with other nitrification inhibitor research throughout the U.S. (Fox \& Bandel, 1989; Frye et al., 1989; Malzer et al., 1989). In well-drained soils of the mid-Atlantic, a nitrification inhibitor did not increase $\mathrm{N}$ fertilizer efficiency or yield (Fox \& Bandel, 1989). However, nitrapyrin has increased yields 22 to $33 \%$ in waterlogged conditions (Ren et al., 2017). DCD has been reported to leach in some instances (McCarty \& Bremner, 1989) while temperature and amount could affect nitrification (Di \& Cameron, 2004). This could affect the efficacy of this nitrification inhibitor, but the leaching capabilities and effects of environmental conditions on the fate of pronitridine have not been reported. In claypan soils, we would expect limited deep leaching, could be affected by the prevalence and intensity of rainfall (Blevins et al, 1996). Differences in decomposition rate of DCD and nitrapyrin based on soil type (Bronson et al., 1989) could affect the crop response observed, but comparisons with pronitridine have not been reported. In a recent meta-analysis of enhanced efficiency fertilizers for corn management systems, nitrapyrin or NBPT plus DCD combined with UAN showed no significant effect $(P=0.84)$ on corn yield data (Cook et al., 2015). However, nitrapyrin and DCD have reduced $\mathrm{N}_{2} \mathrm{O}$ emissions 20 to $90 \%$ (Chen et al., 2010; Di \& Cameron, 2012; Halvorson et al., 2010; Carneiro et al., 2010; Khalil, 2011; Burzaco et al., 2013; Omonode \& Vyn, 2013), which can enhance the efficiency of a UAN application.

\subsection{Protein and Grain N Concentration}

Since $\mathrm{N}$ treatments included a range of amounts, this provided an opportunity to evaluate relationship between grain $\mathrm{N}$ concentration, which was determined chemically, and protein, which was determined with NIR for all treatments in 2013 and 2014. This linear relationship (grain N concentration $=0.1409 \times$ protein concentration) indicated that NIR could provide a good estimate $\left(\mathrm{R}^{2}=0.75\right)$ of grain $\mathrm{N}$ concentration with a more cost-effective, safer, and quicker determination (Figure 2). A standard factor of 6.25 has been used to calculate protein concentration based on $\mathrm{N}$ content in the grain, which can be affected by other $\mathrm{N}$ compounds (FAO, 2003). Grain protein $\mathrm{N}$ content has ranged from 13 to $19 \%$, which has a conversion factor of 0.13 to 0.19 (FAO, 2003) where our conversion factor $(0.1409)$ for corn produced over several $\mathrm{N}$ rates was within this range. Therefore, calculating $\mathrm{N}$ uptake based on protein amount could be a useful tool for evaluating the efficiency of $\mathrm{N}$ treatments, 
especially when protein concentration is determined during harvest (Long et al., 2008). As more data are collected, an improved relationship may be determined over various corn production systems and environments.

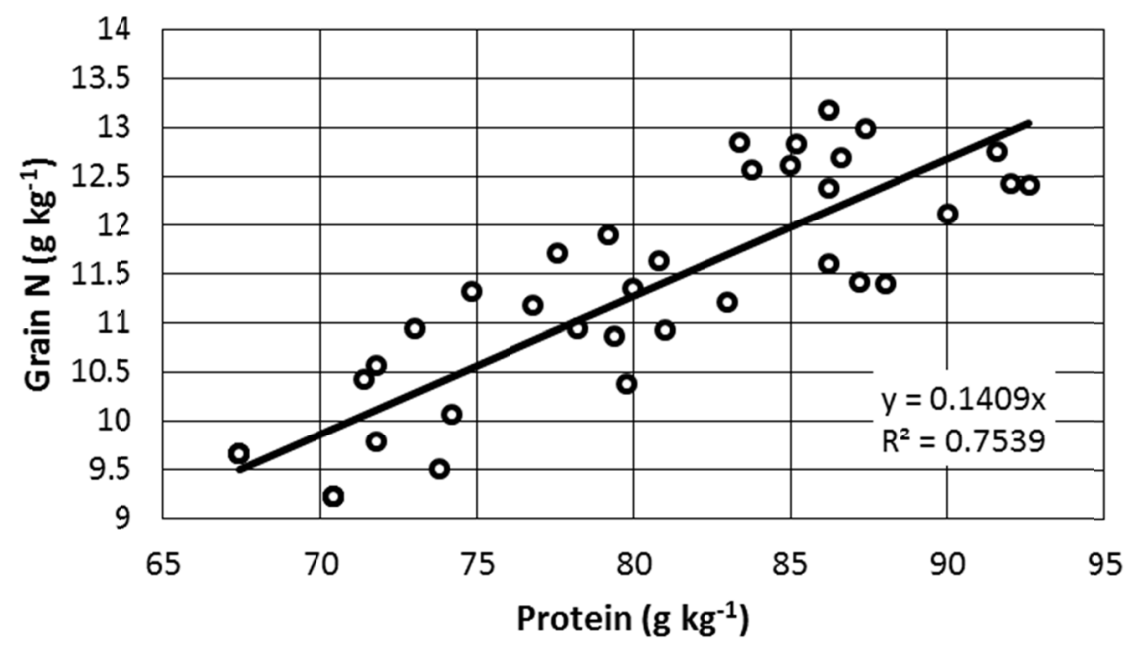

Figure 2. Relationship between corn grain protein concentration and grain $\mathrm{N}$ concentration at Novelty, Missouri. Individual points represent average protein and grain $\mathrm{N}$ concentrations for each $\mathrm{N}$ amount in the presence or absence of a nitrification inhibitor in 2013 and 2014

\section{Conclusions}

In conclusion, corn response to pronitridine and nitrapyrin depended on the $\mathrm{N}$ rate and environmental conditions during the experiments, which affected overall grain yields. In general, urea ammonium nitrate plus pronitridine at $9.4 \mathrm{~L} \mathrm{ha}^{-1}$ affected corn similarly to a higher $\left(18.7 \mathrm{~L} \mathrm{ha}^{-1}\right)$ rate. The amount of additional UAN required to produce yields equivalent to $67 \mathrm{~kg} \mathrm{~N} \mathrm{ha}^{-1}$ plus pronitridine at $9.4 \mathrm{~L} \mathrm{ha}^{-1}$ or nitrapyrin needed to be increased 14 to $19 \%$. Similarly, the amount of nontreated UAN needed to be increased 8 to $11 \%$ in a high yielding year (2014) for yields to be equivalent to UAN at $135 \mathrm{~kg} \mathrm{~N}^{-1}$ plus pronitridine at $9.4 \mathrm{~L} \mathrm{ha}^{-1}$ or nitrapyrin. Grain $\mathrm{N}$ removal and agronomic efficiency was greatest with pronitridine at $9.4 \mathrm{~L} \mathrm{ha}^{-1}$ and nitrapyrin applied with 67 and $135 \mathrm{~kg}$ $\mathrm{N} \mathrm{ha}^{-1}$, respectively, but it was not significantly greater than the other treatments. This research indicates that pronitridine was as effective as nitrapyrin when added to a dribble band of UAN.

\section{Acknowledgements}

The author would like to thank Chris Dudenhoeffer and Lynn Bradley for their technical support and Koch Agronomic Services, LLC for their financial support of this research.

\section{References}

Abendroth, L. J., Elmore, R. W., Boyer, M. J., \& Marlay, S. K. (2011). Corn growth and development. PMR 1009 (p. 50). Ames, Iowa: Iowa State University Extension.

Aita, C., Gonzatto, R., Miola, E. C. C., Santos, D. B., Rochette, P., Angers, D. A., ... Giacomini, S. J. (2014). Injection of dicyandiamide-treated pig slurry reduced ammonia volatilization without enhancing soil nitrous oxide emissions from no-till corn in southern Brazil. Journal Environmental Quality, 43, 789-800. https://doi.org/10.2134/jeq2013.07.0301

Aulakh, M. S., Rennie, A., \& Paul, E. A. (1984). Acetylene and N-Serve effects upon $\mathrm{N}_{2} \mathrm{O}$ emissions from $\mathrm{NH}_{4}{ }^{+}$ and $\mathrm{NO}_{3}{ }^{-}$treated soils under aerobic and anaerobic conditions. Soil Biology \& Biochemistry, 16, 351-356. https://doi.org/10.1016/0038-0717(84)90031-2

Blevins, D., Wilkison, D., Kelly, B., \& Silva, S. (1996). Movement of nitrate fertilizer to glacial till and runoff from a claypan soil. Journal of Environmental Quality, 25(3), 584-593. https://doi.org/10.2134/jeq1996. $00472425002500030026 \mathrm{x}$

Bremner, J. M., \& Blackmer, A. M. (1979). Effects of acetylene and soil water content on emission of nitrous oxide from soils. Nature, 280, 380-381. https://doi.org/10.1038/280380a0 
Bremner, J. M., Breitenbrck, G. A., \& Blackmer, A. M. (1981). Effect of nitrapyrin on emission of nitrous oxide from soil fertilized with anhydrous ammonia. Geophysical Research Letters, 8, 353-356. https://doi.org/ 10.1029/GL008i004p00353

Bronson, K. F., Mosier, A. R., \& Bishnoi, S. R. (1992). Nitrous oxide emissions in irrigated corn as affected by nitrification inhibitors. Soil Science Society of America Journal, 56, 161-165. https://doi.org/10.2136/ sssaj1992.03615995005600010025x

Bronson, K. F., Touchton, K. F., \& Hauck, R. D. (1989). Decomposition rate of dicyandiamide and nitrification inhibition. Communications in Soil Science and Plant Analysis, 20, 2067-2078. https://oi.org/10.1080/ 00103628909368201

Buckley, M. E., Kluitenberg, G. J., Sweeney, D. W., Kelley, K. W., \& Stone, L. R. (2010). Effect of tillage on the hydrology of a claypan soil in Kansas. Soil Science Society of America Journal, 74, 2109-2119. https://doi.org/10.2136/sssaj2010.0024

Burzaco, J. P., Smith, D. R., \& Vyn, T. J. (2013). Nitrous oxide emissions in Midwest US maize production vary widely with band-injected $\mathrm{N}$ fertilizer rates, timing, and nitrapyrin presence. Environmental Research Letters, 8, 1-11. https://doi.org/10.1088/1748-9326/8/3/035031

Carneiro, J., Cardenas, L. M., Hatch, D. J., Trindade, H., Scholefield, D., Clegg, C. D., \& Hobbs, P. (2010). Effect of the nitrification inhibitor dicyandiamide on microbial communities and $\mathrm{N} 2 \mathrm{O}$ from an arable soil fertilized with ammonium sulphate. Environmental Chemistry Letters, 8, 237-246. https://doi.org/10.1007/ s10311-009-0212-3

Cerrato, M. E., \& Blackmer, A. M. (1990). Comparison models for describing corn yield response to nitrogen fertilizer. Agronomy Journal, 82, 138-143. https://doi.org/10.2134/agronj1990.00021962008200010030x

Chen, D., Suter, H. C., Islam, A., \& Edis, R. (2010). Influence of nitrification inhibitors on nitrification and nitrous oxide $\left(\mathrm{N}_{2} \mathrm{O}\right)$ emission from a clay loam soil fertilized with urea. Soil Biology \& Biochemistry, 42, 660-664. https://doi.org/10.1016/j.soilbio.2009.12.014

Cook, R. L., Nail, A. Vigardt, A. Trlica, A., Hagarty, B., Williams, T., \& Wolt, J. (2015). Meta-analysis of enhanced efficiency fertilizers in corn systems in the Midwest. International Plant Nutrition Institute Report. Retrieved from http://research.ipni.net/project/IPNI-2014-USA-4RM06

Delgado, J. A., \& Mosier, A. R. (1996). Mitigation alternatives to decrease nitrous oxides emissions and urea-nitrogen loss and their effect on methane flux. Journal of Environmental Quality, 25, 1105-1111. https://doi.org/10.2134/jeq1996.00472425002500050025x

Di, H. J., \& Cameron, K. C. (2004). Effects of temperature and application rate of a nitrification inhibitor, dicyandiamide (DCD), on nitrification rate and microbial biomass in a grazed pasture soil. Australian Journal of Soil Research, 42, 927-932. https://doi.org/10.1071/SR04050

Di, H. J., \& Cameron, K. C. (2012). How does the application of different nitrification inhibitors affect nitrous oxide emissions and nitrate leaching from cow urine in grazed pastures? Soil Use and Management, 28, 54-61. https://doi.org/10.1111/j.1475-2743.2011.00373.x

Dobermann, A. (2007). Nutrient use efficiency-Measurement and management. Presented at IFA International Workshop on Fertilizer Best Management Practices. Brussels, Belgium.

FAO (Food and Agriculture Organization of the United Nations). (2003). Methods of food analysis. Food energy-Methods of analysis and conversion factors (p. 87). Rome, Italy: FAO.

Fisk, L. M., Maccarone, L. D., Barton, L., \& Murphy, D. V. (2015). Nitrapyrin decreased nitrification of nitrogen released from soil organic matter but not amoA gene abundance at high soil temperature. Soil Biology and Biochemistry, 88, 214-223. https://doi.org/10.1016/j.soilbio.2015.05.029

Fixen, P., Bentrup, F., Bruulsema, T., Garcia, F., Norton, R., \& Zingore, S. (2014). Nutrient/fertilizer use efficiency: Measurement, current situation, and trends. Managing water and fertilizer for sustainable agriculture intensification (pp. 1-30). Paris, France: IFA, IWMI, IPNI, and IPI.

Fox, R. H., \& Bandel, V. A. (1989). Dicyandiamide (DCD) research in agriculture in the mid-Atlantic region. Communications in Soil Science and Plant Analysis, 20, 1957-1968. https://doi.org/10.1080/00103628909 368196

Frame, W. (2017). Ammonia volatilization from urea treated with NBPT and two nitrification inhibitors. Agronomy Journal, 109, 378-387. https://doi.org/10.2134/agronj2016.08.0464 
Francis, D. D., Doran, J. W., \& Lohry, R. D. (1993). Immobilization and uptake of nitrogen applied to corn as starter fertilizer. Soil Science Society of America Journal, 57, 1023-1026. https://doi.org/10.2136/sssaj1993. $03615995005700040024 \mathrm{x}$

Frye, W. W., Graetz, D. A., Locascio, S. J., Reeves, D. W., \& Touchton, J. T. (1989). Dicyandiamide as a nitrification inhibitor in crop production in the southeastern USA. Communications in Soil Science and Plant Analysis, 20, 1969-1999. https://doi.org/10.1080/00103628909368197

Gabrielson, K. D., \& Epling, M. L. (2016). Reaction products and methods for making and using same. US Patent No. 9,440,890 B2. Wichita, KS: Koch Agronomic Services, LLC.

Habibullah, H., Nelson, K. A., \& Motavalli, P. P. (2017). Assessing management of nitrapyrin with urea ammonium nitrate fertilizer on corn yield and soil nitrogen in a poorly-drained claypan soil. Journal of Agronomic Sciences, 9, 17-29. https://doi.org/10.5539/jas.v9n11p17

Halvorson, A. D., Del Grosso, S. J., \& Alluvione, F. (2010). Nitrogen source effects on nitrous oxide emissions from irrigated no-till corn. Journal of Environmental Quality, 39, 1554-1562. https://doi.org/10.2134/ jeq2010.0041

Hughes, T. D., \& Welch, L. F. (1970). 2-Chloro-6-(trichloromethyl) pyridine as a nitrification inhibitor for anhydrous ammonia applied in different seasons. Agronomy Journal, 62, 821-824. https://oi.org/10.2134/ agronj1970.00021962006200060044x

Khalil, M. I. (2011). Physical and chemical manipulation of urea fertilizer to limit the emission of reactive nitrogen species. Understanding Greenhouse Gas Emissions from Agricultural Management ACS Symposium Series (Vol. 1072, pp. 149-164). https://doi.org/10.1021/bk-2011-1072.ch009

Kyveryga, P. M., \& Blackmer, T. M. (2014). Probability of profitable yield response to nitrification inhibitor used with liquid swine manure on corn. Precision Agriculture, 15, 133-146. https://doi.org/10.1007/ s11119-013-9307-8

Long, D. S., Engel, R. E., \& Siemens, M. C. (2008). Measuring grain protein concentration with in-line near infrared reflectance spectroscopy. Agronomy Journal, 100, 247-252. https://doi.org/10.2134/agronj2007. 0052

Malzer, G. L., Kelling, K. A., Schmitt, M. A., Hoeft, R. G., \& Randall, G. W. (1989). Performance of dicyandiamide in the north central states. Communications in Soil Science and Plant Analysis, 20, 2001-2022. https://doi.org/10.1080/00103628909368198

Martin, H. W., Graetz, D. A., Locascio, S. J., \& Hensel, D. R. (1994). Contrasts of nitrapyrin, dicyandiamide, and iso-butylidene diurea effects on total inorganic soil nitrogen. Communications in Soil Science and Plant Analysis, 25, 547-565. https://doi.org/10.1080/00103629409369062

Mason, M. G. (1987). Effects of dicyandiamide (a nitrification inhibitor) on leaching of nitrogen and growth of cereals. Australian Journal of Experimental Agriculture, 27, 127-133. https://doi.org/10.1071/EA9870127

McCarty, G. W., \& Bremner, J. M. (1989). Laboratory evaluation of dicyandiamide as a soil nitrification inhibitor. Communications in Soil Science and Plant Analysis, 20, 2049-2065. https://oi.org/10.1080/ 00103628909368200

Nash, P. R., Motavalli, P. P., \& Nelson, K. A. (2012). Nitrous oxide emissions from claypan soils due to nitrogen fertilizer source and tillage/fertilizer placement practices. Soil Science Society of America Journal, 76, 983-993. https://doi.org/10.2136/sssaj2011.0296

Nash, P. R., Motavalli, P. P., Nelson, K. A., \& Kremer, R. (2015). Ammonia and nitrous oxide gas loss with subsurface drainage and polymer-coated urea fertilizer in a poorly-drained soil. Journal of Soil and Water Conservation, 70, 267-275. https://doi.org/10.2489/jswc.70.4.267

Nathan, M., Stecker, J., \& Sun, Y. (2006). Soil testing in Missouri: A guide for conducting soil tests in Missouri (EC923). Columbia, MO: University of Missouri. Retrieved from http://soilplantlab.missouri.edu/ soil/ec923.pdf

Nelson, K. A., \& Smoot, R. L. (2012). Corn hybrid response to water management practices on claypan soil. International Journal of Agronomy, 2012, Article ID 925408. https://doi.org/10.1155/2012/925408

O’Callaghan, M., Gerard, E. M., Carter, P. E., Lardner, R., Sarathchandra, U., Burch, G., ... Bell, N. (2010). Effect of the nitrification inhibitor dicyandiamide (DCD) on microbial communities in a pasture soil 
amended with bovine urine. Soil Biology \& Biochemestry, 42(9), 1425-1436. https://doi.org/10.1016/ j.soilbio.2010.05.003

Omonode, R. A., \& Vyn, T. J. (2013). Nitrification kinetics and nitrous oxide emissions when nitrapyrin is coapplied with urea-ammonium nitrate. Agronomy Journal, 105, 1475-1486. https://doi.org/10.2134/agronj 2013.0184

Pal, P., McMillan, A. M. S., \& Saggar, S. (2016). Pathways of dicyandiamide uptake in pasture plan. Biology and Fertility of Soils, 52, 539-546. https://doi.org/10.1007/s00374-016-1096-6

Prasad, R., \& Power, J. (1995). Nitrification inhibitors for agriculture, health and the environment. Advances in Agronomy, 54, 233-281. https://doi.org/10.1016/S0065-2113(08)60901-3

Randall, G. W., \& Vetch, J. A. (2003). Corn production on a subsurface-drain Mollisol as affected by time of nitrogen application and nitrapyrin. Agronomy Journal, 95, 1213-1219. https://doi.org/10.2134/agronj 2003.1213

Ren, B., Zhan, J., Dong, S., Liu, P., Zhao, B., \& Li, H. (2017). Nitrapyrin improves grain yield and nitrogen use efficiency of summer maize waterlogged in the field. Agronomy Journal, 109, 185-192. https://doi.org/ 10.2134/agronj2016.06.0353

Ruser, R., \& Schulz, R. (2015). The effect of nitrification inhibitors on the nitrous oxide $\left(\mathrm{N}_{2} \mathrm{O}\right)$ release from agricultural soils-A review. Journal of Plant Nutrition and Soil Science, 178, 171-188. https://doi.org/ 10.1002/jpln.201400251

SAS Institute. (2014). SAS user's guide (v. 9.4). Cary, NC: SAS Inst.

Stelly, M. (1980). Nitrification inhibitors-potentials and limitations. ASA Special Publication 38. Madison, WS: American Society of Agronomy, Soil Science Society of America.

Trenkel, M. E. (2010). Slow- and controlled-release and stabilized fertilizers: an option for enhancing nutrient use efficiency in agriculture (p. 160). Paris, France: International Fertilizer Industry Association.

USDA-ERS (United States Department of Agriculture Economic Research Service). (2015). Fertilizer use and price. Washington, DC: USDA-ERS. Retrieved from https://www.ers.usda.gov/data-products/fertilizer-useand-price

USDM (United States Drought Monitor). (2015). U.S. drought monitor map archive. The National Drought Mitigation Center, Lincoln, NE, USA. Retrieved from http://droughtmonitor.unl.edu/MapsAndData/ MapArchive.aspx

Vetsch, J. A., \& Schwab, G. J. (2014). Corn Grain Yield As Affected By the Nitrification Inhibitor KAS771G77. Presented at the ASA, CSSA, \& SSSA International Annual Meeting. Retrieved from https://scisoc. confex.com/scisoc/2014am/webprogram/Paper86493.html

Weiske, A., Benckiser, G., Herbert, T., \& Ottow, J. C. G. (2001). Influence of the nitrification inhibitor 3,4-dimethylpyrazole phosphate (DMPP) in comparison to dicyandiamide (DCD) on nitrous oxide emissions, carbon dioxide fluxes and methane oxidation during 3 years of repeated application in field experiments. Biology and Fertility of Soils, 34, 109-117. https://doi.org/10.1007/s003740100386

Wilkinson, D. H., Blevins, D. W., \& Silva, S. R. (2000). Use of isotopically labeled fertilizer to trace nitrogen fertilizer contributions to surface, soil, and ground water. Journal of Environmental Hydrology, 8, 1-16.

Wolt, J. D. (2004). A meta-evaluation of nitrapyrin agronomic and environmental effectiveness with emphasis on corn production in the Midwestern USA. Nutrient Cycling in Agroecosystems, 69, 23-41. https://doi.org/ 10.1023/B:FRES.0000025287.52565.99

Zaman, M., Saggar, S., Blennerhassett, J. D., \& Singh, J. (2009). Effect of urease and nitrification inhibitors on $\mathrm{N}$ transformation, gaseous emissions of ammonia and nitrous oxide, pasture yield and $\mathrm{N}$ uptake in grazed pasture system. Soil Biology \& Biochemistry, 41, 1270-1280. https://doi.org/10.1016/j.soilbio.2009.03.011

Zhang, J., Koehler, K. J., Ellsworth, J. W., \& Blackmer, A. M. (2008). Sensitivity of chlorophyll meters for diagnosing nitrogen deficiencies of corn in production agriculture. Agronomy Journal, 100, 543-550. https://doi.org/10.2134/agronj2006.0153

Zurweller, B., Motavalli, P. P., Nelson, K. A., \& Dudenhoeffer, C. J. (2015). Soil nitrous oxide emissions as affected by enhanced efficiency nitrogen fertilizer and temporarily waterlogged conditions. Journal of Agricultural Science, 7(12), 1-14. https://doi.org/10.5539/jas.v7n12p1 


\section{Copyrights}

Copyright for this article is retained by the author(s), with first publication rights granted to the journal.

This is an open-access article distributed under the terms and conditions of the Creative Commons Attribution license (http://creativecommons.org/licenses/by/4.0/). 